\title{
SCH527123, a novel CXCR2 antagonist, inhibits ozone-induced neutrophilia in healthy subjects
}

\author{
O. Holz*, , S. Khalilieh", , A. Ludwig-Sengpiel*, H. Watz*, P. Stryszak\#, P. Soni" \\ M. Tsai" ${ }^{\#}$ J. Sadeh" and H. Magnussen*
}

ABSTRACT: SCH527123 is a novel, selective CXC chemokine receptor 2 antagonist that inhibits neutrophil activation and modulates neutrophil trafficking in animal models, characteristics that may be beneficial in the treatment of conditions with unbalanced pulmonary neutrophilia, such as chronic obstructive pulmonary disease. The purpose of this proof-of-principle study was to determine whether SCH527123 inhibits ozone-induced neutrophil recruitment in healthy humans.

In a randomised, double-blind, placebo-controlled, three-way crossover study, oral SCH527123 (50 mg once daily, 4 days), prednisolone (50 mg once), or placebo was alternated with 2-week washouts. 18 healthy ozone responders ( $>20 \%$ increase in sputum neutrophils) underwent ozone challenge tests ( $250 \mathrm{ppb}, 3 \mathrm{~h}$ intermittent exercise) $1 \mathrm{~h}$ after the last treatment dose. Sputum was induced at $3 \mathrm{~h}$ post-challenge.

After SCH527123 treatment, the ozone challenge resulted in significantly lower sputum neutrophil counts $\left(0.13 \times 10^{6} \cdot \mathrm{mL}^{-1}\right)$ compared with prednisolone $\left(0.84 \times 10^{6} \cdot \mathrm{mL}^{-1} ; p<0.001\right)$ or placebo $\left(2.98 \times 10^{6} \cdot \mathrm{mL}^{-1} ; p<0.001\right)$. Comparable results were obtained for total cell count, percentage of sputum neutrophils, and for interleukin-8 and myeloperoxidase in sputum supernatant. Post-challenge, $\mathrm{SCH} 527123$ inhibited neutrophilia in peripheral blood but significantly less than in sputum. All treatments were safe and well tolerated.

SCH527123 causes significant attenuation of ozone-induced airway neutrophilia in healthy subjects. Further evaluation in a large trial of patients with pulmonary disorders is warranted.

KEYWORDS: Anti-inflammatory, chronic obstructive pulmonary disease, interleukin-8, myeloperoxidase, pharmacodynamics

CH527123 is a novel and selective CXC chemokine receptor 2 (CXCR2) antagonist, which binds CXCR2 on neutrophils, inhibits neutrophil activation, modulates neutrophil trafficking to the CXC chemokines bearing the glutamic acid-leucine-arginine (ELR) motif (i.e., interleukin (IL)-8, growth-related protein (Gro)- $\alpha$, epithelial-derived neutrophil attractant (ENA)78 , neutrophil-activating peptide (NAP)-2), and affects chronic inflammation. Preclinical studies have shown that SCH527123 inhibits pulmonary neutrophilia to various stimuli that increase IL-8 $[1,2]$, suggesting that SCH527123 could play an important role in diseases with excessive pulmonary neutrophilia by restoring the unbalanced immune response.

Chronic obstructive pulmonary disease (COPD) is characterised by airflow limitation that is not fully reversible and is usually progressive [3]. The pathogenesis of COPD involves an inflammatory response to noxious particles and gases such as the chemicals in cigarette smoke, which activate epithelial cells and resident macrophages. These activated cells, in turn, secrete cytokines and chemokines, thereby recruiting neutrophils to the lungs. Activated neutrophils and macrophages secrete proteases that cause alveolar damage, increase mucous secretion, and propagate inflammation. Pulmonary neutrophilia is a major cellular modification within patients with COPD [4-6] and has been associated with a decline in lung function $[3,7,8]$. Therefore, attenuation of neutrophil recruitment and activation may have a positive effect on disease progression and the long-term course of COPD.

\section{AFFILIATIONS}

*Pulmonary Research Institute, Hospital Grosshansdorf, Grosshansdorf, Germany. ${ }^{\#}$ Schering-Plough Research Institute, Kenilworth, NJ, USA.

"Both authors contributed equally to this work.

CORRESPONDENCE

H. Magnussen

Pulmonary Research Institute

Hospital Grosshansdorf

Woehrendamm 80

22927 Grosshansdorf

Germany

E-mail: magnussen@

pulmoresearch.de

Received:

March 252009

Accepted after revision:

July 142009

First published online:

July 302009 
Before testing SCH527123 in patients with chronic neutrophilic airway inflammation, this proof-of-principle study was conducted in healthy subjects in whom airway neutrophilia was induced by the inhalation of ozone. Ozone causes a transient and reproducible increase in sputum neutrophils [9-11], which can be attenuated by short-term therapeutic intervention with both inhaled and oral steroid treatment. Steroids are therefore used as a positive control in this model $[9,12]$. The model has been validated using a moderate dose of ozone $(250 \mathrm{ppb}, 3 \mathrm{~h}$ of intermittent moderate exercise) in healthy volunteer subjects known to be ozone responsive (e.g., minimum increase in sputum neutrophil levels $3 \mathrm{~h}$ after ozone exposure relative to baseline levels [9]). Although acute, short-term, ozone-induced neutrophil recruitment in healthy subjects cannot serve as a COPD disease model, valuable information on the activity of compounds meant to modulate airway neutrophil recruitment can be obtained before proceeding with evaluation of treatment in subjects with chronic neutrophilic airway inflammation.

For a maximum effect size, a dose of $50 \mathrm{mg}$ SCH527123 was chosen. This dose was known from clinical pharmacology studies to be administered safely (blood neutrophil response after single treatment with 10, 50, 100, 150 and $200 \mathrm{mg}$ [13]) and well tolerated in multiple dose treatments (blood neutrophil response during 14 days of treatment with 10, 30 and $50 \mathrm{mg}$ [14]). $50 \mathrm{mg}$ prednisolone was administered only once prior to ozone exposure, as this treatment solely served as a positive control for the ozone challenge model and was not to be compared to the effect of SCH527123.

The objective of this study was to test the hypothesis that SCH527123 inhibits pulmonary neutrophilia, using a previously described ozone-challenge model [9] in healthy subjects. To the authors' knowledge, this is the first test in humans of the pharmacodynamic effects of a CXCR2 antagonist on airway neutrophil recruitment.

\section{MATERIALS AND METHODS Eligibility criteria}

Healthy nonsmoking subjects ( $\leqslant 5$ pack-yrs if ex-smoker) aged $18-55$ yrs, forced expiratory volume in $1 \mathrm{~s}$ (FEV1) $\geqslant 80 \%$ predicted, with normal airway reactivity were eligible to enrol in this study.

\section{Study population}

18 healthy nonsmokers were included in the study (table 1). All subjects had a normal airway response to methacholine (provocative concentration that produces a $20 \%$ fall in FEV1 (PC20) $\geqslant 8 \mathrm{mg} \cdot \mathrm{mL}^{-1}$ methacholine) within $12 \mathrm{months}$ before the study or at screening. The study was approved by the Ethical Committee of the Chamber of Physicians of the State of Schleswig-Holstein, Germany, and all subjects gave written informed consent.

\section{Study protocol}

This single-site, randomised, double-blind, multiple-dose, placebo-controlled, 3-way crossover study (fig. 1) was conducted from November 2004 to June 2005 in conformance with Good Clinical Practices. Subjects were orally administered each of the following treatments on separate occasions in random order: SCH527123, $50 \mathrm{mg}$ once daily for 4 days and single-dose placebo for prednisolone on day 4; placebo for SCH527123, $50 \mathrm{mg}$ once daily for 4 days and single-dose prednisolone $50 \mathrm{mg}$ on day 4; placebo for SCH527123, $50 \mathrm{mg}$ once daily for 4 days and single-dose placebo for prednisolone on day 4. Investigators at the Grosshansdorf site (Pulmonary Research Institute, Grosshansdorf, Germany) enrolled patients. Subject numbers were assigned in the order in which the subjects were enrolled. Randomisation of treatment sequence was accomplished using block randomisation (Williams') design. In total, six different treatment sequences were used: each group of six patients were assigned six sequences in random code order. Treatment was packaged for each patient in a blinded fashion with instructions for administration of numbered containers. The study statistician generated the sequence allocation. The sequence was concealed until interventions were assigned, and all persons involved in the study were blinded to treatment allocation.

During screening, subjects underwent a methacholine challenge test to ensure normal bronchial responsiveness [3, 15] and sputum induction test to ensure their ability to produce sputum in response to hypertonic saline inhalation (baseline sputum). 2 weeks later, eligible subjects underwent an ozone challenge test, followed by sputum induction. Only subjects in whom sputum neutrophils increased at least $20 \%$ over baseline were enrolled.

During the study, subjects underwent an ozone challenge test $1 \mathrm{~h}$ after the last treatment in each treatment period; during the test, subjects inhaled ozone at $250 \mathrm{ppb}$ for $3 \mathrm{~h}$ while exercising at 15 -min intervals. Lung function was measured before exposure, once per hour during exposure, and after exposure, until values returned to within $10 \%$ of the pre-exposure measurement.

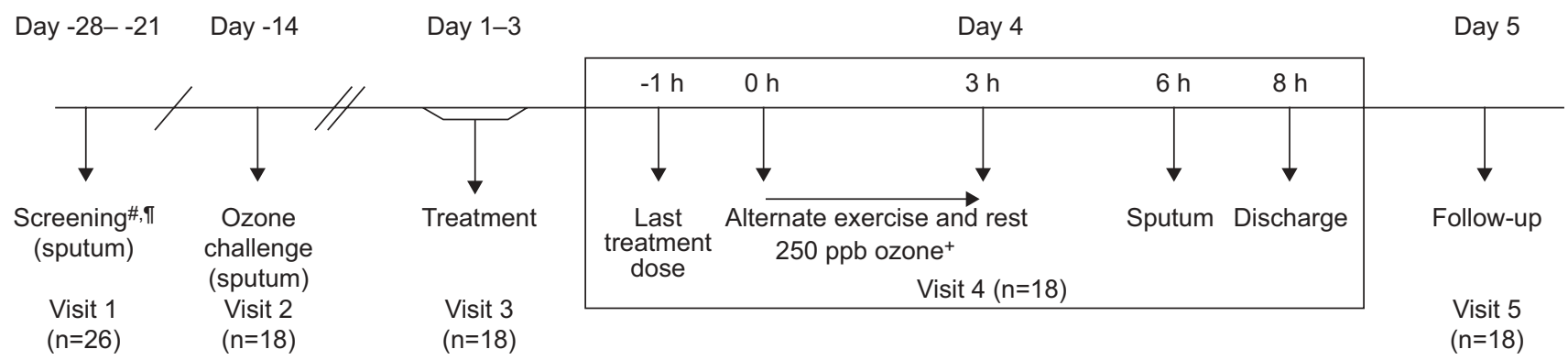

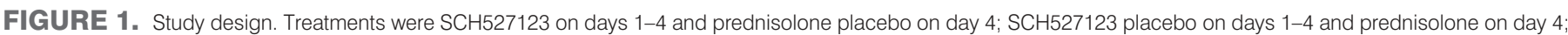

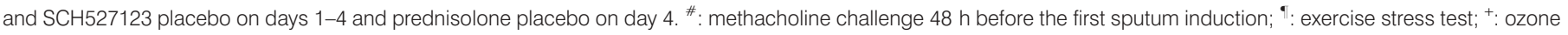
exposures separated by at least 2 weeks. 


\begin{tabular}{lc}
\hline TABLE 1 & Demographics and baseline characteristics \\
\hline Characteristics & Baseline characteristics \\
\hline $\begin{array}{l}\text { Patients } \mathbf{n} \\
\text { Sex }\end{array}$ & $18^{\#}$ \\
$\quad$ Female & $2(11)$ \\
Male & $16(89)$ \\
Race & $17(94)$ \\
White & $1(6)$ \\
$\quad$ Multiracial & $33.3 \pm 12.6$ \\
Age yrs & $24.2 \pm 2.0$ \\
Body mass index $\mathbf{~ k g} \cdot \mathbf{m}^{-\mathbf{2}}$ & $4.344 \pm 0.878$ \\
FEV $\mathbf{~ L ~}$ & \\
\hline
\end{tabular}

Data are presented as $n(\%)$ or mean $\pm S D$, unless otherwise stated. FEV 1 : forced expiratory volume in $1 \mathrm{~s}$. ${ }^{*}$ : one of the 18 participants discontinued at follow-up for prednisolone treatment after experiencing a motor vehicle accident, unrelated to treatment. This participant completed all treatmentrelated procedures, however, and was included in the analysis

Sputum was induced $3 \mathrm{~h}$ after completion of each ozone challenge test. A washout interval of at least 2 weeks separated each treatment period and ozone challenge test $[16,17]$.

\section{Sputum induction and analysis}

Sputum neutrophil counts were the primary outcome; all other sputum parameters were secondary outcomes. Sputum was induced and processed as previously described [10, 18]. Differential cell counts were assessed by two independent, experienced observers from 400 nonsquamous cells and averaged. IL-8 and myeloperoxidase were analysed by ELISA (IL-8: Sanquin, Amsterdam, the Netherlands; myeloperoxidase: Immundiagnostic, Bensheim, Germany).

\section{Peripheral blood neutrophils}

Blood samples were collected for peripheral blood neutrophil counts predose on day 1, day 4 (day of ozone challenge test) pre-dose and at $8 \mathrm{~h}$ post-dose (the projected time of maximum neutrophil suppression), and on day 5 ( $\sim 24 \mathrm{~h}$ following the last dose).

\section{Safety evaluations}

Blood was collected for chemistry and haematology assessments. Safety was also assessed throughout the study by measuring vital signs, monitoring pulmonary function, and assessing adverse events.

\section{Statistical analysis}

This study was powered based on the results of previous ozone challenge studies $[9,19]$. With a sample size of 18 subjects, differences of 0.3 between treatment means in neutrophil counts ( $\log _{10}$ transformed) were estimated to be detectable with $80 \%$ power, assuming a within-subject standard deviation of 0.42 .

All sputum variables were analysed with ANOVA using fixed effects of treatment and period and a random participant effect. Pairwise treatment comparisons, p-values, and 95\% CIs for each pairwise comparison were based on the corresponding ANOVA results. For all sputum parameters (excluding data presented as percentages), the geometric mean and associated 95\% CI were reported.

\section{RESULTS}

\section{Subject disposition and demographics}

26 subjects were screened, but five did not pass the screening criteria because they were unable to produce sputum $(n=2)$ or had intercurrent infection $(n=1)$, high liver enzyme levels $(n=1)$ or a positive methacholine challenge test response $(n=1)$. Two subjects were not randomised because enrolment was complete. One subject withdrew for personal reasons on day 1 of the first treatment. 18 subjects completed all three treatments and sputum-induction procedures (fig. 1, table 1).

\section{Sputum analysis}

All sputum samples were of sufficient quality and were included in the analysis. At baseline (before the ozone challenge test), the geometric mean $(95 \% \mathrm{CI})$ sputum neutrophil count was $0.43(0.23-0.80) \times 10^{6} \cdot \mathrm{mL}^{-1}$. After the screening ozone challenge test, the neutrophil count increased to 3.33 $(2.23-4.95) \times 10^{6} \cdot \mathrm{mL}^{-1} \quad$ (fig. 2). Following treatment with SCH527123, prednisolone or placebo and ozone challenge test, the sputum neutrophil counts were $0.13(0.06-0.23) \times 10^{6} \cdot \mathrm{mL}^{-1}$, $0.84(0.52-1.4) \times 10^{6} \cdot \mathrm{mL}^{-1}$, and $2.98(2.0-4.5) \times 10^{6} \cdot \mathrm{mL}^{-1}$, respectively (fig. 2). Treatment with SCH527123 resulted in a significantly lower mean sputum neutrophil count, compared with prednisolone $(p<0.001)$ and placebo $(p<0.001)$. Mean neutrophil counts after prednisolone treatment were significantly different than in the placebo group $(p=0.001)$. The analysis of sputum neutrophil percentages yielded a similar result (fig. 2). As expected from the observed changes in neutrophil proportions, the percentage of sputum macrophages decreased after the screening ozone challenge test and was higher after SCH527123 or prednisolone, compared with placebo (table 2). The baseline number of macrophages was $2.42(1.55-3.71) \times 10^{6} \cdot \mathrm{mL}^{-1}$, and ozone exposure during screening caused a reduction to $1.62(0.95-2.77) \times 10^{6} \cdot \mathrm{mL}^{-1}$. Macrophage counts were similar after treatment with SCH527123 $\left(1.7 \times 10^{6} \cdot \mathrm{mL}^{-1}\right)$, prednisolone $\left(1.68 \times 10^{6} \cdot \mathrm{mL}^{-1}\right)$, or placebo $\left(1.80 \times 10^{6} \cdot \mathrm{mL}^{-1}\right)$. The effects on total cellularity (fig. 3 ) were comparable to the neutrophil counts. No effects were observed for the number and percentages of sputum eosinophils, lymphocytes, and bronchial epithelial cells.

The treatment effects on sputum differential cell count were confirmed by the analysis of sputum supernatants. As illustrated in figures $4 \mathrm{a}$ and $\mathrm{b}$, changes in sputum myeloperoxidase and IL-8 concentrations after ozone exposure in screening and after treatment with SCH527123, prednisolone, or placebo were comparable to the changes observed for the sputum neutrophil counts.

\section{Peripheral blood neutrophils}

The mean peripheral blood neutrophil count during screening was $3.39 \times 10^{6} \cdot \mathrm{mL}^{-1}$. Inhalation of ozone resulted in an increase in peripheral blood neutrophils $4 \mathrm{~h}$ after the ozone challenge test $(8 \mathrm{~h}$ post-dose), the number in the placebo group was $6.49 \times 10^{6} \cdot \mathrm{mL}^{-1}$. Comparable to the effects observed in sputum, treatment with SCH527123 also reduced the number of peripheral blood neutrophils below the screening value $\left(1.9 \times 10^{6} \cdot \mathrm{mL}^{-1}\right)$. 

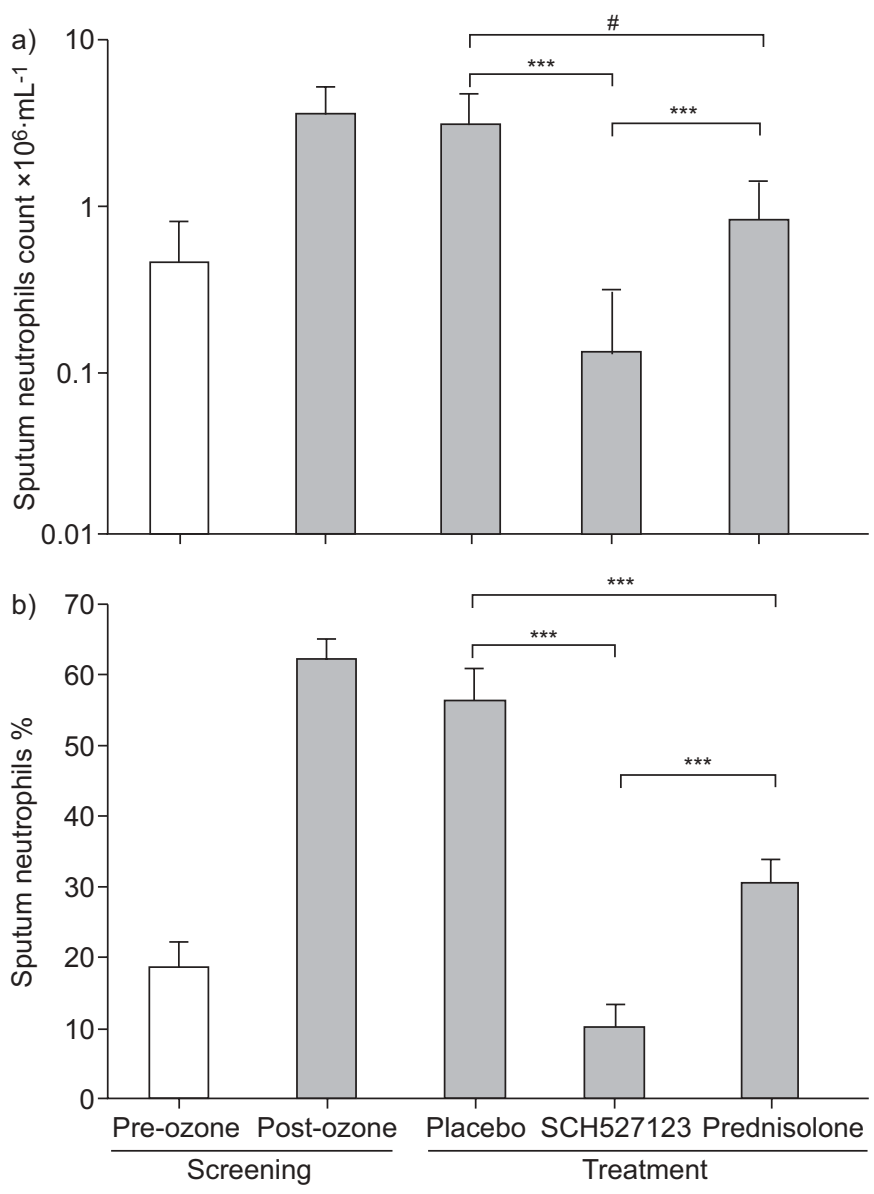

FIGURE 2. a) The geometric mean neutrophil count and b) mean neutrophil percentages and associated $95 \%$ Cls for the screening period (before ( $\square$ ) and after $(\square)$ ozone challenge testing) and each treatment group (after ozone challenge testing). ${ }^{*}: p=0.001 ; * * *: p<0.001$

However, the effect of SCH527123 was smaller in blood than in sputum; treatment with $\mathrm{SCH} 527123$ reduced sputum neutrophil numbers to $4 \%(2.1-8.3 \%)$, whereas blood neutrophil counts remained at $29 \%(24.0-35.5 \%)$ relative to the values measured after placebo ( $\mathrm{p}<0.001$, ANOVA).

\section{Safety}

Adverse events were mild to moderate. The most commonly reported adverse events among patients who received SCH527123 were neutropenia (seven out of 19) and headache (two out of 19). Neutropenia was mild in five cases $(1000 \leqslant$ absolute neutrophil count (ANC) <1500) and moderate in two cases $(500 \leqslant \mathrm{ANC}<1000)$. The latter two cases returned to $>1000$ the day after the last treatment. There were no clinically significant findings in blood pressure, pulse, temperature, electrocardiograms, or clinical safety laboratory analyses. The median (range) decrease from baseline in FEV1 during the ozone challenge test was similar in the SCH527123, placebo, and prednisolone groups: $-6.7 \%(-33.9-7.9 \%),-4.5 \%(-32.4-$ $5.1 \%)$, and $-4.9 \%(-22.4-4.9 \%)$, respectively. There was no significant difference between groups.

\section{DISCUSSION}

This study demonstrated that SCH527123, a novel, selective CXCR2 antagonist, inhibits ozone-induced sputum neutrophilia in healthy subjects by suppressing migration of neutrophils towards CXC chemokines. It is therefore reasonable to expect SCH527123 to inhibit neutrophilia induced by other irritants (e.g., cigarette smoke). Consistently, ozoneinduced increases in sputum total cell count, myeloperoxidase, and IL- 8 were also inhibited. These findings may be relevant to a potential treatment option in COPD through inhibition of excessive neutrophilic airway inflammation, which may play a major role in COPD pathogenesis.

Inhalation of ozone is well known to cause acute pulmonary neutrophilia [11, 12, 16, 19]. The inflammatory response to ozone is transient and shows reproducible differences between subjects [19]. Therefore, the ozone-challenge model is considered to be safe and thus applicable to investigate the pharmacologic modulation of neutrophil traffic and activation. Though not a disease model, this challenge model was selected because the pulmonary inflammatory response to ozone has been well characterised $[9,12,16]$ and mimics some pathologic aspects also observed in COPD, in which a pulmonary irritant (e.g., cigarette smoke) increases IL-8 concentrations, leading to increased pulmonary neutrophil counts. Prednisolone was included in this study as an active control and not for therapeutic comparison to SCH527123 because their mechanisms of action are unrelated.

TABLE 2 Sputum macrophages summary and analysis results (all randomised subjects)

\begin{tabular}{|c|c|c|c|c|c|}
\hline & \multicolumn{2}{|c|}{ Screening } & \multicolumn{3}{|c|}{ Treatment (post-challenge) } \\
\hline Mean $^{\#}$ & 72.89 & 31.97 & 35.14 & 76.28 & 56.28 \\
\hline Median $\#$ & 72.60 & 34.30 & 31.95 & 82.25 & 58.70 \\
\hline $\mathrm{SE}^{\#}$ & 3.55 & 2.50 & 3.78 & 3.98 & 3.55 \\
\hline Prednisolone-placebo & & & & & $20.51(31.33-9.69)^{\star \star \star \star}$ \\
\hline
\end{tabular}

Data are presented as $\%$ or mean difference $(95 \% \mathrm{Cl})$, unless otherwise stated. ${ }^{\#}$ : unadjusted (raw) values; " : based on ANOVA crossover model with period, treatment, and participant effects (pooled SD: 15.86); ${ }^{* *}: \mathrm{p}<0.001$. 


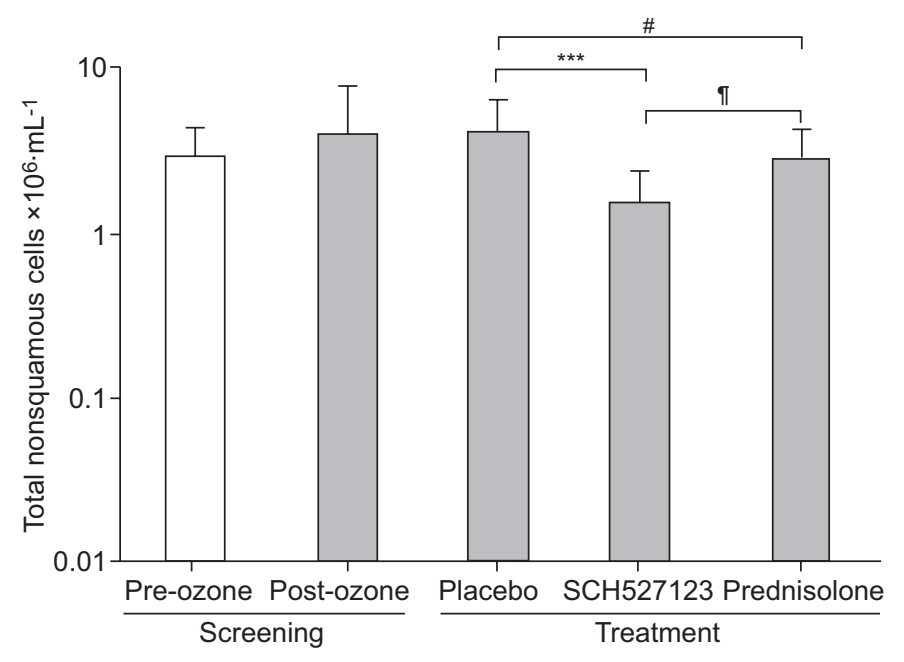

FIGURE 3. The effect of ozone on total sputum cellularity at screening and after treatment with $\mathrm{SCH} 527123$, prednisolone, or placebo. Data are presented as geometric mean with associated $95 \% \mathrm{Cls}$, before $(\square)$ and after $(\square)$ ozone challenge testing. ${ }^{* * *}: p<0.001 ;{ }^{*}: p=0.003 ;{ }^{\natural}: p=0.067$.

However, the effect of prednisolone was comparable to that which was initially described and demonstrates the reproducibility of the ozone-challenge model $[9,12]$.

Age-related loss in lung function is accelerated in patients with COPD [3] and thought to be driven by cycles of inflammation and repair, with subsequent obliteration of small airways as well as ventilation-perfusion mismatching and loss in the alveolar gas exchange surface [20]. Inflammation and repair are, in part, stimulated by the inhalation of pulmonary irritants, such as cigarette smoke, pollution and ozone. The irritants activate pulmonary macrophages that secrete cytokines, such as IL-8 and Gro- $\alpha$, which attract and activate neutrophils [3, 21]. The neutrophils recruited to the site of inflammation release enzymes, such as elastase, lysozymes and myeloperoxidase [3], and microcidal superoxides are generated at the cell surface of the neutrophils [22]. In addition, neutrophils themselves release IL-8. Therefore, these mediators released by neutrophils not only result in excessive tissue damage but also propagate the cycle of inflammation. Furthermore, neutrophil elastase has been implicated in goblet cell hyperplasia, which contributes to mucus hypersecretion [3-6]. Increased mucus production can create a pulmonary environment prone to bacterial colonisation and infection, leading to COPD exacerbations. Therefore, druginduced reduction of increased numbers of neutrophils may be considered a potential new treatment option in COPD. Conversely, the global role of neutrophils is broad and complex, and although in excess they can contribute to pulmonary tissue damage, neutrophils help perform important functions, including immune defense and initiation of repair processes [23].

Currently, no therapy for COPD addresses the underlying pathophysiology of the disease. Only the cessation of cigarette smoking has been found to slow the decline of pulmonary function. Bronchodilators such as anticholinergics and $\beta$ agonists, which are first-line therapies for COPD, only alleviate symptoms. Multiple studies have shown that bronchodilators have little or no effect on the accelerated loss in lung function,
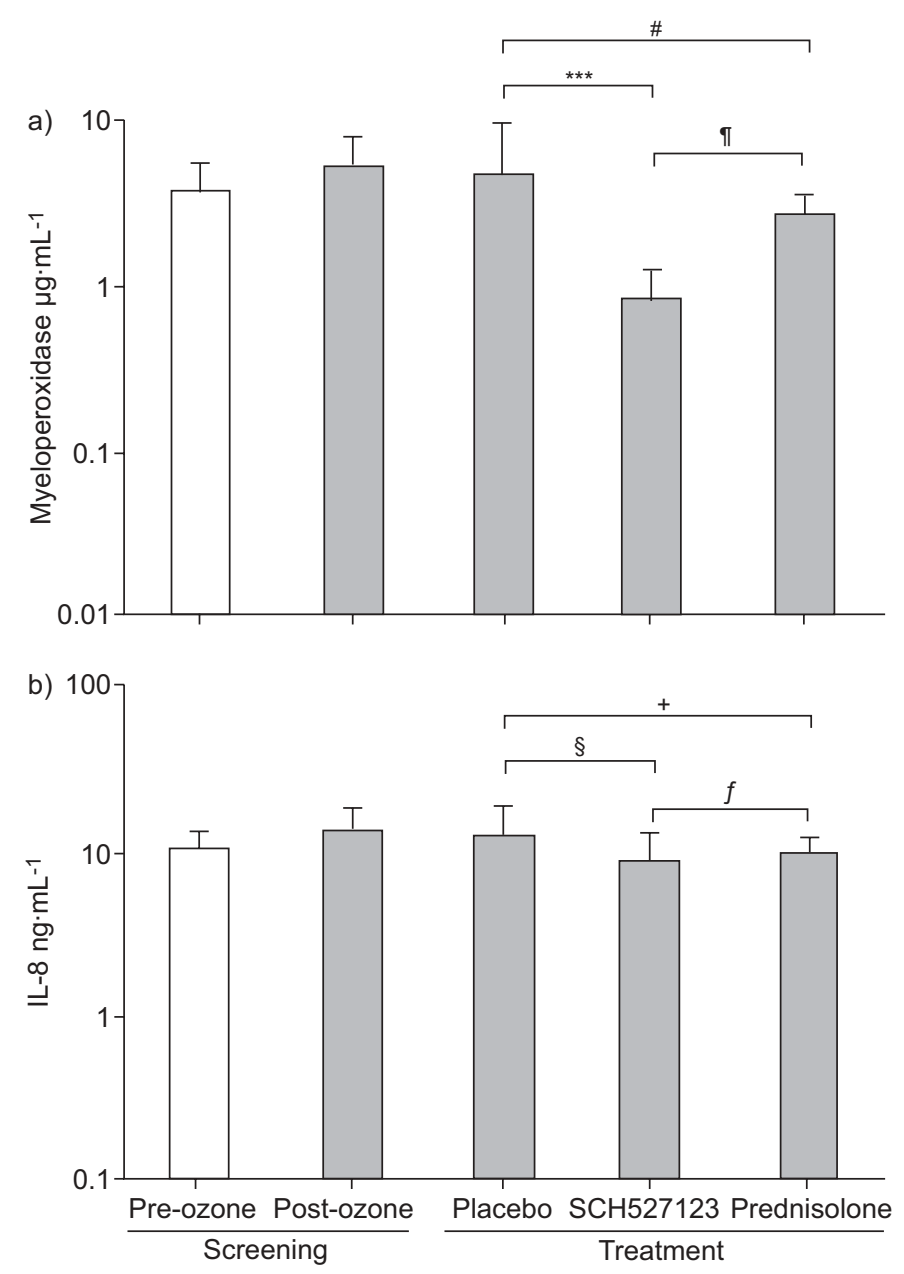

FIGURE 4. The geometric mean sputum concentrations of a) myeloperoxidase and b) interleukin (IL)-8 and associated 95\% Cls for the screening period (before ( $\square$ ) and after $(\square)$ ozone challenge testing) and each treatment group (after ozone challenge testing). ${ }^{* *}: p<0.001 ;{ }^{*}: p=0.025 ;{ }^{\bullet}: p=0.004 ;{ }^{+}: p=0.106 ;{ }^{\S}: p=0.028$; $f: p=0.531$

the key cause of death in COPD [3]. Other drugs aimed at decreasing the inflammatory process in the lungs, such as phosphodiesterase- 4 inhibitors, have had a minimal response and are associated with significant side effects. Inhaled corticosteroids, the principal anti-inflammatory therapy in asthma, have only minor effects on the inflammation in COPD and their clinical effects on lung function, symptoms and exacerbations are small [3].

The anti-inflammatory effects of corticosteroids require the action of histone deacetylases, which suppress the expression of inflammatory genes. It was recently reported that histone deacetylase-2 is reduced in peripheral lung and macrophages of patients with COPD [24] and that this is mediated by oxidative stress [25]. Therefore, based on the results of the current study, SCH527123 therapy in patients with COPD may lower oxidative stress by reducing the pulmonary neutrophil count and may restore sensitivity to corticosteroids.

A reversible decrease in peripheral blood neutrophil counts also was observed in the current study, consistent with results 
of previous studies $[13,14]$. This effect may be related to the putative pharmacology of SCH527123; the CXCR2 antagonism toward neutrophils is hypothesised to reversibly inhibit neutrophils' egress from the bone marrow and movement from peripheral blood to tissue. This hypothesis is based on preclinical study results in which a reversible increase in mature neutrophils in bone marrow was observed [26]. It is speculated that when SCH527123 binds to CXCR2 on mature neutrophils in the bone marrow, the balance of cell surface expression may shift from predominant CXCR2 to CXCR4 [27, 28]. When CXCR4 interacts with its ligand, stromal cellderived factor (SDF)-1 $\alpha$, neutrophils are retained in the bone marrow [29], which is thought to be the reason for the decrease observed in peripheral blood neutrophils. This balance toward CXCR2 is restored with administration of granulocyte colonystimulating factor, which inhibits SDF- $1 \alpha$. In a previous study [13], the reduction in peripheral blood neutrophils was immediately reversed after administration of granulocyte colony-stimulating factor, which is consistent with the selective mechanism of SCH527123 (i.e., SCH527123 does not block neutrophil activation and migration to non-ELR CXC chemokines). Another hypothesis is that neutrophils marginate after administration of SCH527123. Clinical studies are ongoing to further understand the mechanism of the reversible decrease in peripheral blood neutrophils. We would like to emphasise that the dose of $50 \mathrm{mg}$ SCH527123 used in this study was chosen to maximise the effect size in this prove of concept study. Future clinical trials in patients will use lower doses to find the optimal dose range for this compound, which is capable of restoring a balanced pulmonary neutrophilia, e.g. in COPD, without causing blood neutropenia.

In this study, inhalation of ozone also causes peripheral blood neutrophilia. However, relative to placebo, SCH527123 inhibited sputum neutrophilia more than it did peripheral blood neutrophilia. This suggests that SCH527123 may be more selective in inhibiting neutrophilia in the target tissue than in the peripheral blood. A similar selectivity could be stated for prednisolone (data not shown). However, the results are confounded by the finding that prednisolone causes peripheral blood neutrophilia through demargination and inhibition of apoptosis [30].

In the current study, all treatments were well tolerated. Aside from the reversible decrease in peripheral blood neutrophil count, no other effects on haematology or chemistry parameters were seen. None of the treatments affected vital signs, electrocardiograms or FEV1, and adverse events were similar among the treatment groups. Despite this, trials of longer duration must be conducted to exclude effects on the immune system in patients with COPD.

In summary, the novel and selective CXCR2 antagonist SCH527123 caused a clear attenuation of ozone-induced airway neutrophilia in healthy human subjects. Therefore, SCH527123 could be a novel treatment option for COPD, in which increased neutrophil numbers play a role in the disease process. However, given the redundancy of the immune system, the efficacy of SCH527123 in patients with COPD will depend on the extent of damage caused by other inflammatory cells and must be evaluated in a large trial of patients with COPD.

\section{SUPPORT STATEMENT}

This research was funded by Schering-Plough Research Institute.

\section{STATEMENT OF INTEREST}

A statement of interest for S. Khalilieh, P. Strysak, P. Sorii, M. Tsai and J. Sadeh, and for the study itself, can be found at www.erj.ersjournals. $\mathrm{com} / \mathrm{misc} /$ statements.dtl

\section{ACKNOWLEDGEMENTS}

The authors would like to thank all subjects who participated in this study; the Environmental Protection Agency of Hamburg, Germany, for its help in gas calibration; and S. Janicki (Pulmonary Research Institute, Grosshansdorf, Germany) for excellent technical assistance.

\section{REFERENCES}

1 Chapman RW, Minnicozzi M, Celly CS, et al. A novel, orally active CXCR1/2 receptor antagonist, Sch527123, inhibits neutrophil recruitment, mucus production and goblet cell hyperplasia in animal models of pulmonary inflammation. J Pharmacol Exp Ther 2007; 322: 486-493.

2 Thatcher TH, McHugh NA, Egan RW, et al. Role of CXCR2 in cigarette smoke-induced lung inflammation. Am J Physiol Lung Cell Molec Physiol 2005; 289: L322-L328.

3 Rabe KF, Hurd S, Anzueto A, et al. Global strategy for the diagnosis, management, and prevention of chronic obstructive pulmonary disease: GOLD executive summary. Am J Respir Crit Care Med 2007; 176: 532-555.

4 Keatings VM, Collins PD, Scott DM, Barnes PJ. Differences in interleukin- 8 and tumor necrosis factor- $\alpha$ in induced sputum from patients with chronic obstructive pulmonary disease or asthma. Am J Respir Crit Care Med 1996; 153: 530-534.

5 Rutgers SR, Postma DS, ten Hacken NH, et al. Ongoing airway inflammation in patients with COPD who do not currently smoke. Thorax 2000; 55: 12-18.

6 Stockley RA. Neutrophils and the pathogenesis of COPD. Chest 2002; 121: 151S-155S.

7 Stanescu D, Sanna A, Veriter C, et al. Airways obstruction, chronic expectoration, and rapid decline of FEV1 in smokers are associated with increased levels of sputum neutrophils. Thorax 1996;51: 267-271.

8 O'Donnell RA, Peebles C, Ward JA, et al. Relationship between peripheral airway dysfunction, airway obstruction, and neutrophilic inflammation in COPD. Thorax 2004; 59: 837-842.

9 Holz O, Tal-Singer R, Kanniess F, et al. Validation of the human ozone challenge model as a tool for assessing anti-inflammatory drugs in early development. J Clin Pharmacol 2005; 45: 498-503.

10 Holz O, Jörres RA, Koschyk S, et al. Changes in sputum composition during sputum induction in healthy and asthmatic subjects. Clin Exp Allergy 1998; 28: 284-292.

11 Balmes JR, Chen LL, Scannell C, et al. Ozone-induced decrements in FEV1 and FVC do not correlate with measures of inflammation Am J Respir Crit Care Med 1996; 153: 904-909.

12 Alexis NE, Lay JC, Haczku A, et al. Fluticasone propionate protects against ozone-induced airway inflammation and modified immune cell activation markers in healthy volunteers. Environ Health Perspect 2008; 116: 799-805.

13 Khalilieh S, Tsai M, de Vries D, et al. Rising single-dose safety and pharmacokinetics of oral SCH 527123, a novel antagonist of CXCR2, in healthy volunteers. Eur Respir J 2007; 30: Suppl. 51, 613s.

14 Khalilieh S, Tsai M, van Marle S, et al. Rising multiple-dose safety and pharmacokinetics of oral SCH 527123, a novel antagonist of CXCR2, in healthy smokers. Eur Respir J 2007; 30: Suppl. 51, 612s.

15 Jörres RA, Nowak D, Kirsten D, et al. A short protocol for methacholine provocation testing adapted to the Rosenthal-Chai dosimeter technique. Chest 1997; 111: 866-869. 
16 Jörres RA, Holz O, Zachgo W, et al. The effect of repeated ozone exposures on inflammatory markers in bronchoalveolar lavage fluid and mucosal biopsies. Am J Respir Crit Care Med 2000; 161: 1855-1861.

17 Holz O, Mücke M, Paasch K, et al. Repeated ozone exposures enhance bronchial allergen responses in subjects with rhinitis or asthma. Clin Exp Allergy 2002; 32: 681-689.

18 Pin I, Gibson PG, Kolendowicz R, et al. Use of induced sputum cell counts to investigate airway inflammation in asthma. Thorax 1992; 47: 25-29.

19 Holz O, Jörres RA, Timm P, et al. Ozone-induced airway inflammatory changes differ between individuals and are reproducible. Am J Respir Crit Care Med 1999; 159: 776-784.

20 Stockley RA. Neutrophils and protease/antiprotease imbalance. Am J Respir Crit Care Med 1999; 160: S49-S52.

21 Adams DH, Lloyd AR. Chemokines: leucocyte recruitment and activation cytokines. Lancet 1997; 349: 490-495.

22 van Beurden WJ, Wielders PL, Scheepers PJ, et al. Superoxide production by peripheral polymorphonuclear leukocytes in patients with COPD. Respir Med 2003; 97: 401-406.

23 Nathan C. Neutrophils and immunity: challenges and opportunities. Nature Reviews Immunology 2006; 6: 173-182.
24 Ito K, Ito M, Elliott WM, et al. Decreased histone deacetylase activity in chronic obstructive pulmonary disease. $N$ Engl J Med 2005; 352: 1967-1976.

25 Barnes PJ. Reduced histone deacetylase in COPD: clinical implications. Chest 2006; 129: 151-155.

26 Evans E, Pastino G, Mirro EJ. Preclinical safety profile of oral $\mathrm{SCH}$ 527123, a novel antagonist of CXCR2. Eur Respir J 2007; 30: Supp1. $51,775 \mathrm{~s}$.

27 Suratt BT, Petty JM, Young SK, et al. Role of the CXCR4/SDF-1 chemokine axis in circulating neutrophil homeostasis. Blood 2004; 104: 565-571.

28 Martin C, Burdon PC, Bridger G, et al. Chemokines acting via CXCR2 and CXCR4 control the release of neutrophils from the bone marrow and their return following senescence. Immunity 2003; 19: 583-593.

29 Semerad CL, Liu F, Gregory AD, et al. G-CSF is an essential regulator of neutrophil trafficking from the bone marrow to the blood. Immunity 2002; 17: 413-423.

30 Mann BS, Chung KF. Blood neutrophil activation markers in severe asthma: lack of inhibition by prednisolone therapy. Respir Res 2006; 7: 59 . 